\title{
Vivências de acadêmicos de medicina na promoção da I Oficina de Contagem de Carboidratos para crianças e adolescentes portadores de Diabetes Mellitus tipo 1
}

\author{
Deborah Laredo Jezinia*, Michelle Masuyo Minami Sato ${ }^{\mathrm{b}}$, Marcela Figueiredo \\ Conceição Azevedo ${ }^{\mathrm{a}}$, Andrezza Mendes Franco $^{\mathrm{c}}$, Vitória Miki Pang Takatani ${ }^{\mathrm{c}}$, Priscila \\ Picanço Horta ${ }^{\mathrm{c}}$, Rosevelto Maia Borges ${ }^{\mathrm{c}}$, Fábio Lucas Silva Fernandes ${ }^{\mathrm{c}}$ \\ aDepartamento de Clínica Médica, Universidade Federal do Amazonas (UFAM), Manaus, AM, Brasil \\ ${ }^{\text {b} R e s i d e ̂ n c i a ~ M e ́ d i c a ~ d o ~ H U G V ~-~ U F A M, ~ U n i v e r s i d a d e ~ F e d e r a l ~ d o ~ A m a z o n a s ~(U F A M), ~ M a n a u s, ~ A M, ~ B r a s i l ~}$ \\ ${ }^{\mathrm{c}}$ Curso de Medicina, Universidade Federal do Amazonas (UFAM), Manaus, AM, Brasil
}

\section{Histórico do Artigo Recebido em: 10/01/2020 \\ Aceito em: \\ $15 / 03 / 2020$}

\section{Palavras-chave:}

Diabetes mellitus tipo 1; terapia nutricional; contagem de carboidratos; criança; adolescente; aprendizagem

Keywords: Diabetes mellitus type 1; nutrition therapy; carbohydrate Counting; Children; Adolescents; Learning

\section{RESUMO}

O Diabetes Mellitus tipo I (DM1) é uma doença autoimune caracterizada pela perda progressiva da capacidade de produção e secreção de insulina pelas células beta-pancreáticas. Acomete principalmente crianças e adolescentes e corresponde a 5-10\% de todos os casos de Diabetes Mellitus. O tratamento empregado envolve um manejo delicado da glicemia por meio da administração de insulina exógena, dos hábitos alimentares e prática de atividades físicas. A Contagem de Carboidratos é incluída neste contexto como uma ferramenta importante para obtenção do equilíbrio e flexibilidade nas escolhas alimentares e na manutenção do perfil glicêmico adequado. O objetivo desse estudo foi relatar as experiências vividas pelos acadêmicos de medicina na promoção de uma oficina prática de contagem de carboidratos junto ao público infanto-juvenil. Sob orientação de uma equipe multidisciplinar da área da saúde, realizou-se uma atividade acadêmica de extensão e de interação com a comunidade em que se optou por utilizar uma abordagem teórico-prática: iniciando com palestras introdutórias ministradas por profissionais da área; seguida por exercícios práticos de contagem de carboidratos com os pacientes, simulando situações reais do cotidiano tendo os acadêmicos como monitores. Os estudantes puderam atuar em todas as etapas de planejamento e execução da oficina; auxiliar os pacientes durante as atividades práticas foi um grande aprendizado, uma vez que eles interagiram bastante, sanaram dúvidas e puderam consolidar os conhecimentos adquiridos anteriormente. É possível constatar que as vivências dos acadêmicos em eventos desse gênero fortalecem o aprendizado adquirido em sala de aula, desenvolvendo também princípios éticos e morais necessários para o bom profissional da medicina, contribuindo para a uma formação acadêmica diversificada e focada na atenção integral ao paciente.

Experiences of medicine academics in the promotion of the I Carbohydrate Counting Workshop for children and adolescents with Type 1 Diabetes

\begin{abstract}
Diabetes Mellitus type I (DM1) is an autoimmune disease characterized by the progressive loss of insulin production and secretion capacity by beta-pancreatic cells. It mainly affects children and adolescents and corresponds to 5-10\% of all cases of Diabetes Mellitus. The treatment employed involves a delicate handling of blood glucose through the administration of exogenous insulin, eating habits and physical activity. Carbohydrate Counting is included in this context as an important tool for achieving balance and flexibility in food choices and maintaining the appropriate glycemic profile. The objective was to report on the experiences lived by medical students in promoting a practical carbohydrate counting workshop with children and young people.Under the guidance of a multidisciplinary health team, an academic activity of extension and interaction with the community was carried out, in which a theoretical-practical approach was chosen: starting with introductory lectures given by professionals in the area; followed by practical carbohydrate counting exercises with patients, simulating real everyday situations with academics as monitors. Students were able to act in all stages of planning and executing the workshop; helping patients during practical activities was a great learning experience, since they interacted a lot, resolved doubts and were able to consolidate the knowledge acquired previously. It is possible to verify that the experiences of academics in events of this kind strengthen the learning acquired in the classroom, also developing ethical and moral principles necessary for the good professional of medicine, contributing to a diversified academic formation and focused on comprehensive care to the patient.
\end{abstract}

\footnotetext{
*Autor correspondente: dljezini @gmail.com (Jezini D.L.)
} 


\section{Introdução}

O Diabetes Mellitus tipo 1 (DM1) é uma doença endocrinológica de natureza autoimune, cuja fisiopatologia é centrada na destruição imunomediada das células beta do pâncreas endócrino, que culmina na redução progressiva da produção e secreção de insulina (1). Acerca da epidemiologia, esse tipo de DM equivale a 5 a $10 \%$ da totalidade dos casos, sendo mais prevalente na população infanto-juvenil (2). No Brasil, a estimativa é que o DM1 acometa cerca de 30 mil indivíduos, segundo a International Diabetes Federation (IDF) (3); quando é avaliada a distribuição dos casos de acordo com a região brasileira, as regiões norte e nordeste juntas abrigam 30,8\% dos casos de DM1 do país, ficando atrás apenas da região sudeste com 39,3\% (4).

O DM1 pode ser classificado como autoimune ou tipo 1A, que corresponde a maioria dos casos, e tipo 1B ou idiopático, por não tem participação de autoanticorpos na fisiopatogenia (5).

A predisposição genética é um ponto importante para a fisiopatogenia do DM1, principalmente devido a presença dos genes do sistema HLA de classe 2 (dois), DR3, DR4, PTPN22 e CTLA4 (6). Os gatilhos ambientais, a exemplo de infecções virais, são determinantes para desencadear a resposta imunológica, gerada a partir da infiltração de neutrófilos, macrófagos e linfócitos $\mathrm{T}$, com a produção de autoanticorpos (anticorpo anti-ilhota, anti-insulina, anti-tirosino-fosfatase e anti-GAD), responsáveis pela promoção do processo que culmina com a destruição progressiva das células beta da ilhota de Langherans e o consequente comprometimento na produção de insulina (7). A deficiência absoluta de insulina que se estabelece, desencadeia o quadro clinico, geralmente de forma súbita, com sintomas clássicos: poliúria (excesso de diurese), polidipsia (excesso de sede), polifagia (excesso de fome), perda de peso, entre outros, sendo este grupo mais propenso a desenvolver a cetoacidose diabética (30\%) (8). O tratamento por sua vez é individualizado e tem como base a administração de insulina exógena para obtenção de níveis glicêmicos adequados, associada à prática de exercício físico e alimentação saudável (9).

Entretanto, manter um controle adequado da doença não é uma tarefa simples, uma vez que ao longo do dia ocorrem oscilações significativas da glicemia, sendo necessária a monitorização glicêmica em intervalos regulares a fim de saber a dose correta de insulina a ser administrada, caso contrário pode desencadear quadros de hipoglicemia e hiperglicemia severos, necessitando inclusive de suporte hospitalar especializado, podendo também causar prejuízo no desenvolvimento neuropsicomotor desses indivíduos (10).

Dentro desse universo, a Contagem de Carboidratos (CC) desponta como um instrumento importante no âmbito da manutenção de uma alimentação saudável, proporcionando uma maior flexibilidade ao paciente com relação aos alimentos ingeridos e as doses de insulina necessárias para supri-las, além de oferecer uma melhor adaptação ao estilo de vida de cada paciente, contribuindo para o sucesso do tratamento (11). Esta ferramenta terapêutica utiliza cálculos envolvendo a quantidade total de carboidratos presentes nos alimentos que serão ingeridos em uma refeição, o fator de sensibilidade do paciente ao tipo de insulina a ser aplicada e a meta glicêmica desejada (12). Para isso, é necessária uma avaliação por uma equipe multidisciplinar (1), em especial por profissionais nutricionistas para definir a quantidade de carboidratos que deve ser ingerida diariamente para cada pessoa, levando em consideração o estilo de vida, a dieta, a prática de atividades físicas e o fator de sensibilidade $(13,14)$.

O objetivo deste trabalho é relatar a experiência de estudantes de medicina pertencentes a uma liga acadêmica em uma intervenção junto a portadores de DM1 da faixa etária infanto-juvenil abordando a prática da contagem de carboidratos. 


\section{Materiais e Métodos}

\section{Planejamento e Organização:}

\section{Projeto de Extensão - responsáveis:}

Como atividade de extensão da Universidade Federal do Amazonas (UFAM), os professores de Endocrinologia do Departamento de Clínica Médica (DCM) foram os responsáveis pelo planejamento e organização da "I Oficina Prática de Contagem de Carboidratos", que incluiu a participação de Endocrinologistas adulto e pediátrico, nutricionistas voluntários e de 20 estudantes componentes da Liga Universitária de Metabologia e Endocrinologia (LUME); a motivação foi a dificuldade relatada pelos pacientes portadores de DM tipo $1 \mathrm{em}$ implementar a contagem de carboidratos no tratamento durante as consultas na unidade.

\section{Prévia do Projeto:}

As reuniões de planejamento incluíram os endocrinologistas, a equipe de nutrição; e posteriormente, os alunos voluntários que elaboraram a dinâmica da oficina em conjunto. A equipe de nutrição propôs que as estações representassem situações do dia a dia, onde os pacientes e responsáveis simulariam a contagem, incluindo a possibilidade de escolher entre as opções de alimentos.

Uma semana antes da Oficina, houve um treinamento com os alunos voluntários; a equipe de Nutrição, além de repassar a parte teórica da contagem de $\mathrm{CH}$ e manejo da hipoglicemia, realizou simulações com o propósito de preparar os alunos que seriam tutores dos pacientes.

\section{Seleção dos participantes:}

A seleção dos 41 participantes foi, inicialmente, por meio de convite ao grupo de portadores de DM1, cadastrados no Ambulatório de Endocrinologia geral e pediátrica do Ambulatório Araújo Lima (AAL) ligado ao Hospital Universitário Getúlio Vargas (HUGV/UFAM/EBSERH); e, a confirmação mediante preenchimento de formulário online, cujo endereço eletrônico foi divulgado no ambulatório e nas redes sociais da LUME. Após o preenchimento de todas as vagas disponibilizadas, alguns pacientes ficaram em lista de espera.

O limite de idade para participar da Oficina foi de 23 anos, a fim de contemplar indivíduos mais velhos, que igualmente necessitavam da intervenção. Não houve limite inferior de idade do paciente para inscrição, sendo o participante mais jovem com dois anos de idade. Os pacientes menores de idade eram acompanhados obrigatoriamente por um responsável, já determinado no ato da inscrição; a ideia do projeto era conscientizar inclusive a família no auxílio da escolha dos alimentos e na contagem de $\mathrm{CH}$.

\section{Montagem das estações de simulação nutricional:}

Foram definidas seis estações de simulação nutricional; na montagem das estações de simulação foram de acordo com as refeições do dia e momentos especiais, sendo divididas em duas para o café-da-manhã, duas para o almoço e duas para as festas infantis e comemorações; assim, as dúvidas foram bem especificas para cada momento, tendo como apoio os profissionais tutores e alunos responsáveis pelas estações. 
Os alunos da LUME levaram os alimentos reais dos cardápios e entre as opções saudáveis e não, era necessário pelo menos um alimento em embalagem para criar oportunidade de ensinar sobre como ler corretamente os rótulos.

Os cardápios utilizados nas estações foram montados pelos nutricionistas, com ajuda dos alunos, incluindo frutas, cereais, hortaliças, proteínas, leite e derivados, gorduras e óleos, açúcares e doces.

Os grupos de alimentos escolhidos para cada estação foi diversificado, com opções saudáveis e outras não, incluindo alimentos ricos em açúcar; se optou também por adicionar em todas as estações, opções típicas da culinária amazonense, para tornar cada situação mais próxima da realidade do dia a dia dos pacientes e seus responsáveis.

\section{Material de apoio utilizado nas estações nutricionais:}

Com as estações prontas, o passo seguinte foi escolher o material de apoio. Além dos alimentos, foram necessárias balanças (digital eletrônica de precisão SF-400 1g a 10 $\mathrm{kg}$ ), e para cada uma das seis estações, duas balanças digitais de bolso mini Xtpox modelo b500 de $0,1 \mathrm{~g}$ até $500 \mathrm{~g}$, material de papelaria para pacientes e responsáveis, utensílios da cozinha para ensinar sobre quantidades (medidas caseiras) e a tabela de CC. A Sociedade Brasileira de Diabetes disponibilizou para o evento exemplares impressos do Manual de CC (2016) que pode ser distribuída aos 41 pacientes inscritos.

\section{Data e local do evento:}

O evento foi realizado no dia 22/06/2019 no auditório de um hospital público, parceira da LUME, em formato de oficina prática e abordagem em grupo de pacientes e familiares. A referência base utilizada foi o Manual de Contagem de Carboidratos $(\mathrm{CH})$ para pessoas com Diabetes, do Departamento de Nutrição da Sociedade Brasileira de Diabetes de 2016 (12), que inclui a Tabela de Alimentos.

\section{Dinâmica da Oficina:}

\section{Apresentação do conteúdo teórico:}

No dia da Oficina, a programação começou com apresentação teórica expositiva, com uso de slides com linguagem acessível e interação com a plateia, sendo as aulas ministradas pelas equipes médica e de nutrição; onde os pacientes tiveram a oportunidade de aprender sobre diversos temas que foram abordados, como os métodos de contagem de $\mathrm{CH}$, efeito dos alimentos na variação da glicemia, conhecimento dos alimentos potencialmente ricos em carboidrato, tipos de $\mathrm{CH}$, índice glicêmico, como agir diante da hipoglicemia, conhecer as medidas empregadas (tamanho das colheres, copos e xícaras), entre outros.

\section{Distribuição de grupos e distribuição de resolução de casos de CC:}

Divididos em grupos aleatoriamente, os participantes inicialmente receberam um "paciente fictício", com fator de sensibilidade à insulina (FSI), dose de insulina e glicemia anterior pré-determinados para facilitar a correção dos cálculos.

Conforme orientação dos endocrinologistas, os "pacientes fictícios" das diferentes estações deveriam apresentar glicemias variadas, vivência de situações de hipoglicemia, normoglicemia e hiperglicemia.

Os grupos fizeram rodízio entre as estações, de modo que todos puderam vivenciar 
cada uma das situações propostas pelas diferentes estações.

Oficina na prática:

Para a parte prática, os acadêmicos foram divididos em trios, que organizavam os alimentos com tabelas onde constavam as quantidades de $\mathrm{CH}$, assim como as balanças, em suas respectivas mesas e os pacientes foram divididos entre os diferentes cenários. Os participantes receberam o material de apoio educativo impresso (manual de CC), as dúvidas restantes eram sanadas pelas endocrinologistas e pelas nutricionistas, ambos tutores; ao final, alguns dos pacientes deram depoimentos sobre o aprendizado adquirido na oficina.

\section{Resultados e discussão}

Os acadêmicos tiveram a oportunidade de participar de todas as etapas do evento, desde a idealização, organização da oficina e um treinamento interno com a nutricionista, até a coordenação das equipes que se revezavam entre os diferentes cenários, reforçando o espírito de trabalho em equipe dentro do ambiente de uma atividade acadêmica extracurricular de extensão voluntária.

Ademais, a experiência de interagir com pacientes das mais diversas idades foi muito enriquecedora, uma vez que a imersão no cotidiano desses pacientes, proporcionou um aprendizado prático mais aprofundado sobre a doença como a convivência com ela se faz no dia a dia, demonstrando que a relação médico-paciente e a atenção integral são pilares para o sucesso da terapêutica designada ao paciente.

Os participantes da oficina relataram que as explanações iniciais foram cruciais para que pudessem compreender melhor os exercícios de contagem de carboidratos, vividos nas estações e inclusive, reforçou os conceitos aprendidos por aqueles que já utilizam esse método dentro de seus esquemas terapêuticos.

Eles também receberam material educativo impresso, a exemplo do Manual de Contagem de Carboidratos e das tabelas nutricionais disponibilizadas pela Sociedade Brasileira de Diabetes, e também foram apresentados a aplicativos que auxiliam na contagem da quantidade de carboidratos existentes nas refeições e no cálculo da dose de insulina a ser aplicado.

No momento prático da oficina, os pacientes tiveram a chance de interagir com os acadêmicos e outras pessoas na mesma condição de saúde, compartilhando experiências, além de poder exercitar e fixar melhor os conceitos aprendidos com simulações de situações reais, utilizando medidas caseiras e alimentos reais, sanando as dúvidas no momento em que elas surgiram e percebendo que apesar de trabalhosa, a contagem de carboidratos é uma ferramenta possível de ser usada e que pode contribuir muito com o tratamento, especialmente por conferir mais flexibilidade quanto às escolhas e horários das refeições.

\section{Considerações finais}

A realização da I Oficina de Contagem de Carboidratos foi uma atividade de extensão e interação com portadores de DM1; importante para esclarecer pontos chaves dessa terapia e principalmente, para incentivar os pacientes não adeptos ao método, por considerarem complicado; que, a partir dos ensinamentos, passaram a perceber as vantagens da Contagem de Carboidratos desde que seja com orientação adequada. 
Por outro lado, os acadêmicos puderam aprofundar os conhecimentos adquiridos em sala de aula na preparação para auxiliar os pacientes nas estações, com o suporte de uma equipe multidisciplinar; exercitar o trabalho em equipe; e constatar que a Educação em Diabetes, a atenção integral ao paciente e a uma relação médico-paciente saudável são questões fundamentais para assegurar uma boa adesão ao tratamento e uma melhor qualidade de vida aos pacientes.

\section{Referências}

1. Sociedade Brasileira de Diabetes. Diabetes na prática clínica e-book 2.0 - Módulo 3 - Capítulo 5. [Internet]. São Paulo, 2014; Disponível em: http://www.diabetes.org.br/ebook/component/k2/ item/50-a-educacao-em-diabetes-e-a-equipe-multiprofissional

2. Maraschin JF, Murussi N, Witter V, Silveiro SP. Classificação do diabete melito. Arq Bras Cardiol 2010; 95(2): 40-7.

3. International Diabetes Federation. IDF Diabetes Atlas [Internet]. $9^{\mathrm{a}}$ ed. Bruxelas (BEL): IDF; 2019. Availablefrom: http:// www.idf.org/diabetesatlas

4. Souza ACCB. Avaliação da qualidade de vida de pacientes com Diabetes Mellitus tipo 1: dados do primeiro estudo multicêntrico no Brasil. 2013.

5. Sociedade Brasileira de Diabetes. Diretrizes da Sociedade Brasileira de Diabetes, 2014-2015 / 20152016.

6. Silva MER, Mory D, Davini E. Marcadores genéticos e auto-imunes do diabetes melito tipo 1: da teoria para a prática. Arquivos Brasileiros de Endocrinologia \& Metabologia 2008; 52 (2): 166-180.

7. De Sousa AA, Albernaz AC, Sobrinho HMR. Diabetes Melito tipo 1 autoimune: aspectos imunológicos. Universitas: Ciências da Saúde 2016; 14(1): 53-65, 2016.

8. Maruichi MD, Takamune DM, Noronha, RM, Schechtman, HP, Belhaus MS, Kochi C, Monte O, Calliari LEP. Características de crianças e adolescentes portadores de Diabetes Mellitus tipo 1 ao diagnóstico. Comparação entre dois períodos com dez anos de diferença em serviço universitário. Arquivos Médicos dos Hospitais e da Faculdade de Ciências Médicas da Santa Casa de São Paulo 2018; 57(2): 55-58.

9. Diretrizes da Sociedade Brasileira de Diabetes 2017-2018 / Organização José Egídio Paulo de Oliveira, Renan Magalhães Montenegro Junior, Sérgio Vencio. - São Paulo: Editora Clannad, 2017.

10. Almeida $\mathrm{AB}$ et al. Métodos de Tratamento para Diabetes Mellitus Tipo 1: uma Revisão. International Journal of Nutrology 2018; 11 (S 01): 532.

11. Care, Diabetes. Foundations of Care: Education, Nutrition, Physical Activity, Smoking Cessation, Psychosocial Care, and Immunization. Sec. 4. In Standards of Medical Care in Diabetes-2015. Diabetes Care 2015; S41-S48.

12. Manual de Contagem de Carboidratos para pessoas com Diabetes / Sociedade Brasileira de Diabetes, Departamento de Nutrição. 2016; (Disponível em https://www.diabetes.org.br/publico/materiaispara-download).

13. Bertin RL, Elizio NPS, Moraes RNT, Medeiros CO, Fiori LS, Ulbrich AZ. Percepções do cotidiano alimentar de crianças e adolescentes com diabetes mellitus tipo 1. Revista Contexto \& Saúde 2016; 16(30): 100-109.

14. Smart CE, Annan F, Bruno LPC, Higgins LA, Acerini CL. Nutritional management in children and adolescents with diabetes. Pediatric Diabetes 2014; 15 (Suppl. 20): 136-154. 